\title{
IRAC Deep Survey Of COSMOS
}

\author{
Nick Scoville*, Peter Capak*, Mauro Giavalisco ${ }^{\dagger}$, Dave Sanders**, Lin \\ Yan $^{*}$, Herve Aussel ${ }^{\S}$, Olivier Ilbert ${ }^{* *}$, Mara Salvato*, Bahram Mobasher ${ }^{\star}$ \\ and Emeric LeFloc'h** \\ ${ }^{*}$ California Institute of Technology, MC 105-24, Pasadena, CA 91125, USA \\ ${ }^{\dagger}$ Department of Astronomy, University of Massachusetts, LGRT-B 520,710 N. Pleasant St., \\ Amherst, MA 01003, USA \\ ${ }^{* *}$ Institute for Astronomy, University of Hawaii, 2680 Woodlawn Dr., Honolulu, HI 96822, USA \\ ${ }^{\ddagger}$ Spitzer Science Center, Caltech MC 220-6, Pasadena, CA 91125, USA \\ ${ }^{\S}$ CNRS, CEASSacloy, Bat. 709, Gif-sur-Yvette, 91191, France \\ "Department of Physics and Astronomy, UC Riverside, Riverside, CA, 92521, USA
}

\begin{abstract}
Over the last four years, we have developed the COSMOS survey field with complete multi-wavelength coverage from radio to X-ray, including a total of 600 hours of Spitzer Legacy time ( 166 hours IRAC, 460 hours MIPS). Here we propose to deepen the IRAC $3.6 \mu \mathrm{m}$ and $4.5 \mu \mathrm{m}$ coverage with 3000 hours over $2.3 \mathrm{deg}^{2}$ area included in deep Subaru imaging. This extended mission deep survey will increase the sensitivity by a factor of 3-5. The most important impact will be that the COSMOS survey will then provide extremely sensitive photometric redshifts and stellar mass estimates for approximately a million galaxies out to $z \sim 6$. We expect these data to detect approximately 1000 objects at $z=6$ to 10 . The data will also provide excellent temporal coverage for variability studies on timescales from days to the length of the extended mission.
\end{abstract}

Keywords: Spitzer Space Telescope, infrared astronomical observations, origin and formation of universe, external galaxies, distances, large scale structure

PACS: $95.85 . \mathrm{Hp}, 98.62 . \mathrm{Py}, 98.62 . \mathrm{Ve}, 98.80 . \mathrm{Bp}$

\section{COSMOS OVERVIEW}

The COSMOS survey probes the coevolution of galaxies, AGN and cosmic large scale structures (LSS). The 2 square degree field samples scales of 30-180 Mpc at $\mathrm{z}=0.2-4-$ including a million galaxies in a volume of over $10^{7} \mathrm{Mpc}^{3}$, similar to SDSS in the local universe (Scoville et al. [1]). Star formation, galactic stellar masses and morphologies and AGN are sampled with greatly reduced cosmic variance as a function of redshift over the full range of environments at each epoch.

\section{THE UNIQUE COSMOS DATASET}

The Cosmic Evolution Survey (COSMOS) was the largest project ever undertaken on the Hubble telescope. During the years 2003 and 2004, 10\% of the Hubble's time was devoted to imaging a 2 degree area of the sky on the celestial equator with ACS. The COSMOS Spitzer survey also is one of the two largest Spitzer Legacy programs with 650 hours over two cycles (Sanders et al. [2]). A major CXO survey (Elvis et al. in prep.) with 1.8 million seconds (Ms) is being done this year, building upon the $1.6 \mathrm{Ms}$

\footnotetext{
CP943, The Science Opportunities for the Warm Spitzer Mission Workshop, edited by L. J. Storrie-Lombardi and N. A. Silbermann

(C) 2007 American Institute of Physics 978-0-7354-0457-1/07/\$23.00
} 
project already completed with XMM (Hasinger et al. [3]). Major investments have also been made by virtually every large ground-based telescope- most importantly 35 nights with Subaru for broad band imaging to yield photometric redshifts (Taniguchi et al. [4], Mobasher et al. [5]) and 540 hours with the VLT for spectroscopy (Lilly et al. [6]). These comprehensive datasets coordinated and made public through the COSMOS team are enabling major studies of evolution for both the luminous and dark matter at $\mathrm{z}>0.5$ to 5 . Over a million galaxies are seen in these data - by far the largest sample ever studied in the early universe.

An important feature of COSMOS is the development of complete, very sensitive, multi-wavelength datasets. Deep optical/infrared imaging (Subaru, CFHT, and NOAO) provides photometric redshifts (photo-z with $\sigma_{z} /(1+z) \simeq 0.02$, see Fig. 1) and SEDs for $\sim 700,000$ galaxies at $I_{\mathrm{AB}}<26 \mathrm{mag}$, enabling the tracing of LSS in the galaxies. $\mathrm{XMM}$ and $\mathrm{CXO}$ probe the densest clusters in diffuse X-ray emission (and also detect 3000 AGN point-sources). Weak lensing analysis of the HST-ACS imaging reveals the dark matter LSS. SFRs and AGN measures are derived independently from the IR (Spitzer), UV (GALEX), radio (VLA) and X-ray (XMM/CXO) observations. Initial results including source counts and sensitivities for the S-COSMOS Legacy programs in IRAC and MIPS are presented in Sanders et al. [2].
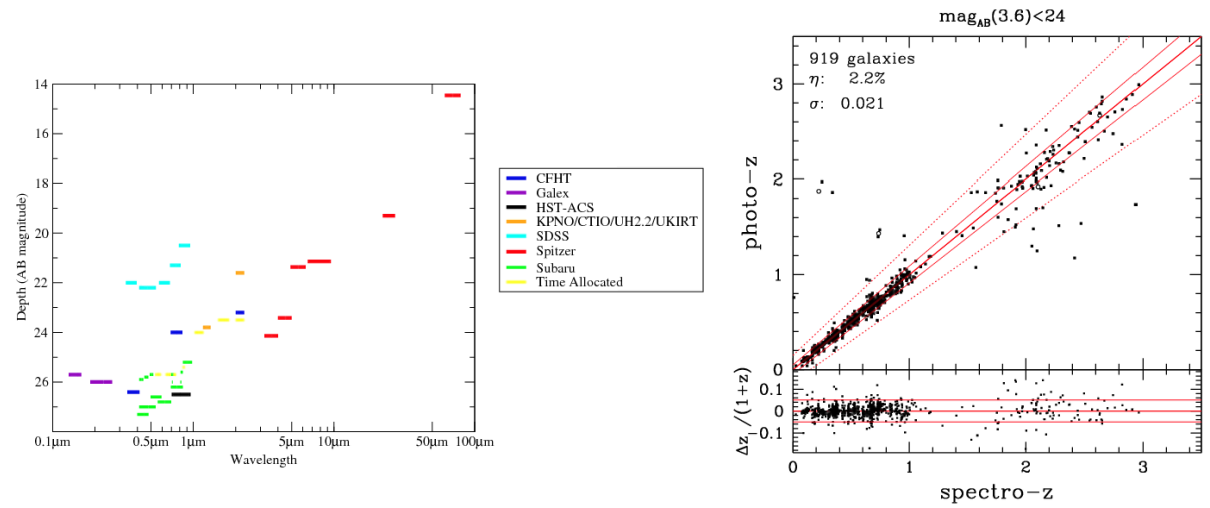

FIGURE 1. Left - The 5- $\sigma$ sensitivities are shown for the UV-optical and IR bands in COSMOS (3 arcsec apertures except ACS 0.15 arcsec) Capak et al. [7]. Right - Comparison of the newly derived COSMOS photo-z's using 20 bands (Ilbert et al. in prep.) with the spectroscopic redshifts (Lilly et al. [6]) for 2100 galaxies with $3.6 \mu \mathrm{m}<24 \operatorname{mag}_{A B}$ (some with $I_{\mathrm{AB}}$ down to $26 \mathrm{mag}$ ) indicates $\sigma_{z} /(1+z)<0.02$.

\section{LARGE SCALE STRUCTURE IN COSMOS}

A major goal of COSMOS has recently been realized and was a highlight of the Jan'07 AAS meeting and a Nature cover (see Fig. 2) - the first imaging of large scale structures (38 LSS on scales of 3-30 Mpc at $\mathrm{z}=0.2-1.1$ ) in both the dark matter from weak lensing (Massey et al. [8]) and in the baryons from galaxy overdensities (Scoville et al. [9], Guzzo et al. [10]) and diffuse X-ray emission (Hasinger et al. [3], Finoguenov et al. [11]). The dependence of morphology and galactic spectral energy distribution (SED) 
on environment has been followed in these structures and into the field population (Scoville et al. [9], Capak et al. [12]). Large structures have also been seen in COSMOS at $\mathrm{z} \sim 3-4.5$ in Lyman Break Galaxies (LBG: Lee et al. in prep.) and at $\mathrm{z}=5.7$ in Ly $\alpha$ emitting sources Murayama et al. [22]).

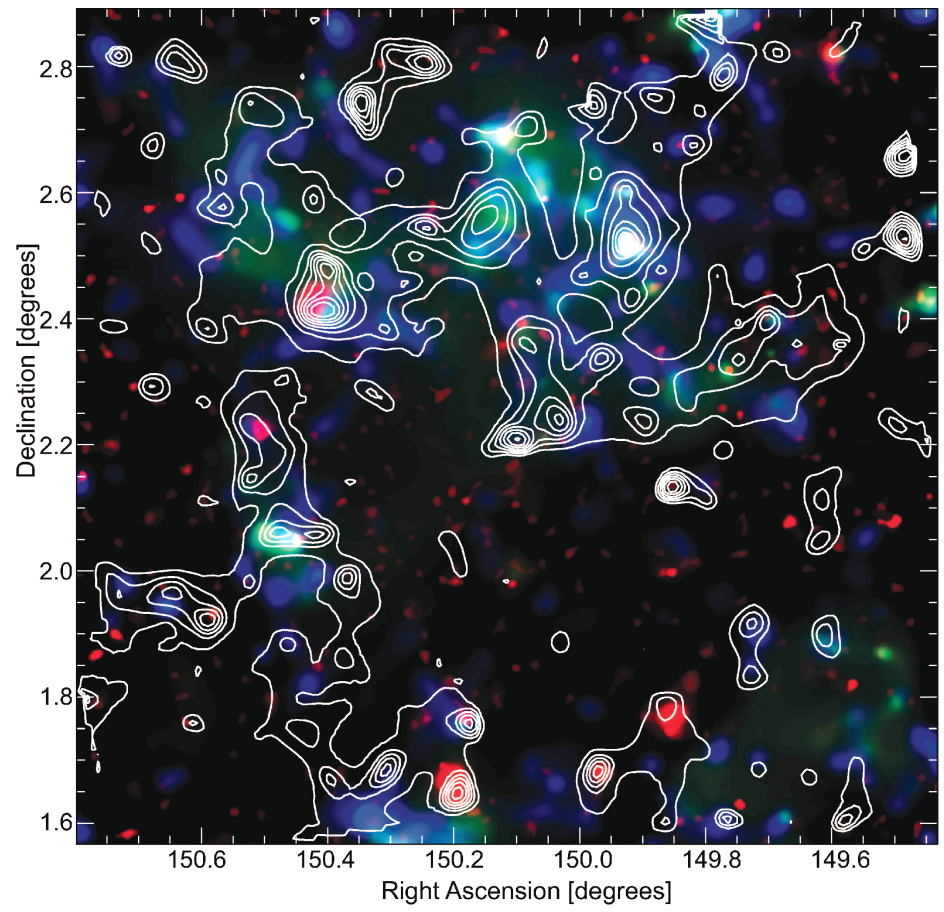

FIGURE 2. The large structures of dark matter (contours, Massey et al. [8]) and baryons (colored background, Scoville et al. [9]) are shown for the COSMOS area of the sky. The blue color indicates the concentrations of galaxies and the red shows the hot X-ray emitting gas (Finoguenov et al. [11]) at the centers of the most dense galaxy clusters. The largest filaments can be tracked over $\sim 30 \mathrm{Mpc}$ in the dark matter and the galaxies.

\section{COSMOS ULTRA-DEEP IRAC}

Ultra-deep IRAC observations as proposed here will be a very significant advance over earlier IRAC COSMOS data, enabling:

- detection and sampling of the galaxy mass function down to few $\times 10^{10} M_{\odot}$ (less than the Milky Way) out to $\mathrm{z} \sim 7$;

- very deep IRAC data for the large COSMOS field will provide an unparalleled sample of high redshift galaxies $(z>0.5)$ with masses and SED types for precision galaxy evolution studies with respect to both environment and redshift; 
- photometric redshifts at $1.5<z<5$ with very high accuracy $\left(\sigma_{z} /(1+z)<0.02\right)$. The present optical photometry catalog detects over a million objects at $\left(I_{A B}<26\right)$ while the present IRAC depth detects only 300,000 . The greater depth proposed here should yield IRAC detections of virtually all of the optically detected objects

- greatly improving their photo-z's and stellar mass estimates.

Precision redshifts are essential for the definition and tracing of large scale structure - in COSMOS the photometric redshifts are of sufficiently high accuracy to enable large scale structure studies at $\mathrm{z}<1.1$. (The large scale structures shown in Fig. 2 were at $\mathrm{z}<1.1$.) A major goal of the deep COSMOS IRAC observations is to provide sufficiently deep and uniform near infrared data that the photo-z's can be extended out to $\mathrm{Z} \sim 5$ with sufficient accuracy to enable large scale structure investigations at the earlier epochs spanning the peak of galaxy and AGN evolution. The COSMOS team has proven experience in the derivation of very high accuracy photo-z, combining the IRAC data with the COSMOS ancillary imaging.

Compared with other survey fields, COSMOS offers much more extensive panchromatic ancillary data and the very large COSMOS field cover the full range of large scale structure environment and has greatly reduced cosmic variance at each redshift.

\subsection{Sensitivities}

There are two 38.9 day windows a year when COSMOS is observable and the moon is far enough away from the field. If we assume a 2 year mission that works out to 3734 hours. If we assume $2 / 3$ of the time with low zody background we can observe COSMOS for 2500 hours. In the present data we get 1200 seconds of integration time per pointing for 166 hours of real time. Scaling that we will reach 2.5 hours per pointing per year. The expect sensitivities for the $3.6 \mu \mathrm{m}$ and $4.5 \mu \mathrm{m}$ IRAC exposures are given in Table 1, using the backgrounds actually encountered in previous COMOS IRAC observations. For comparison, we also include in this table the depths obtained for GOOD IRAC. The last column assumes that the extended mission might extend to 2014!

TABLE 1. IRAC Sensitivities $-5 \sigma(\mathrm{AB})$

\begin{tabular}{|c|c|c|c|c|}
\hline$\lambda_{\mu m}$ & pres. $(0.3 \mathrm{hr} / \mathrm{pt})$ & $2.5 \mathrm{hr} / \mathrm{pt}=1 \mathrm{yr}$ & $7.5 \mathrm{hr} / \mathrm{pt}=3 \mathrm{yr}$ & $12.5 \mathrm{hr} / \mathrm{pt}=5 \mathrm{yr}$ \\
\hline 3.6 & 24.0 & 25.2 & 25.7 & 26 \\
\hline 4.5 & 23.3 & 24.4 & 25.0 & 25.4 \\
\hline total time & 166 hours & 1250 hours & 4750 hours & 6250 hours \\
\hline$\lambda_{\mu m}$ & GOODS deep & GOODS ultra-deep & & \\
\hline 3.6 & 26.4 & 27.2 & & \\
\hline 4.5 & 25.6 & 26.4 & & \\
\hline total time & 23 hours & 100 hours & & \\
\hline
\end{tabular}




\subsection{The Necessity For Deep Rest-frame NIR}

The major science drivers for deeper IRAC coverage of the COSMOS field are :

- Understanding the buildup of stellar mass in galaxies during the critical epoch of galaxy assembly and peak star formation at $\mathbf{z}=1$ to 3 . The IRAC $3.6 \mu \mathrm{m}$ and $4.5 \mu \mathrm{m}$ coverage probes the restframe near infrared to visible - the ultra deep coverage proposed here will yield detections and photometrically-derived mass estimates for galaxies down to a few $\times 10^{10} M_{\odot}$ in this redshift range, well below the knee in the mass function. And at $\mathrm{z}<1.5$, the greatest evolution for the early type galaxies is in the lower mass systems; these will be now detected in the deeper IRAC data.

- Much higher reliability photometric redshifts for galaxies at $z>1$ where the limited sensitivity of the existing COSMOS near infrared data compromises the accuracy. Higher sensitivity near infrared data will help break degeneracies in the photo-z fitting. As noted earlier, the deeper IRAC data should enable detections of nearly a million objects, compared with 300,000 detected now.

- Detection of very large samples of objects at $z>6$ to 10 . Although few if any objects have yet been detected and verified at $z>6$, recent studies (e.g. Haiman and Loeb [13], Yahata et al. [14], Yan et al. [15]) indicate that over 1000 objects could be detected at these redshifts with the improved sensitivity over the large COSMOS field.

- Characterization of the population of high-z IR-luminous sources invisible from the optical and near-IR windows. IRAC will be the most efficient (and unique way) to determine which are massive starbursts, which are power-law dominated AGNs, as well as to pin down their accurate localization (in preparation of their follow-ups with NIRCAM and MIRI on JWST and with ALMA).

- These data obtained on repetition timescales spanning from days to the maximum extent of the Spitzer mission (as great as 9 years) will enable major investigations of variable sources such as AGN and stars.

- Lastly, we note that the additional IRAC data with many more exposures on each pointing can be programmed with a dither pattern to optimally reach the theoretical Spitzer 3.6 $\mu \mathrm{m}$ PSF FWHM = 1.44 arcsec). This could be extremely helpful for sources which are somewhat blended in the existing IRAC images.

Very little is known about high redshift $(z>3)$ galaxies because they are faint and relatively rare. Also, the region of the galaxy Spectral Energy Distribution (SED) containing mass and age information is redshifted to the near and mid infrared where it is more difficult to measure. Deep IRAC observations of COSMOS complement the extremely deep observations in GOODS and UDF by probing the bright end and knee of the luminosity and mass function at $z>3$ as well as probing a large enough volume to reduce cosmic variance. Importantly, the objects in COSMOS are bright enough to allow for detailed studies, not possible with the fainter samples. Furthermore, the VLT spectroscopic program will provide redshifts many of the Spitzer-detected objects between $1.5<z<4$. 
The COSMOS data is amongst the best ever collected for studying large scale structure at high redshift. Over 60,000 galaxies are found at $z>3$ in COSMOS. These data represent a factor of 10 improvement in our knowledge of the $z>3$ universe. The largest high redshift survey to date (Steidel et al. [16]) provides 2347 galaxies brighter than $I<25.5$ at $2.5<z<3.5$, of which 940 are spectroscopically confirmed. In contrast, COSMOS contains $\sim 34,000$ galaxies brighter than $I<25.5$ at $2.5<z<3.5$ of which over 9,000 will be spectroscopically confirmed. The deep 3.6 and $4.5 \mu \mathrm{m}$ imaging of COSMOS provided by the warm mission will probe a magnitude below $L_{*}$ at $z \sim 3$ (Steidel et al. [17]) and to $L_{*}$ at $z=6$ (Iwata et al. [18]), providing an unprecedented census of the high redshift universe.

At $z>5$ the universe is approximately a billion years old, providing very little time for galaxies to form and evolve. Yet, several studies show large structures were already visible (Hu et al. [19, 20], Ouchi et al. [21], Murayama et al. [22]) and some massive, $\left(10^{10} M_{\odot}\right)$ relatively old $(400 \mathrm{Myr})$ galaxies were already in place at these redshifts (Mobasher et al. [23], Eyles et al. [24, 25], Yan et al. [26, 15], Lai et al. [27]). Detailed studies of these first galaxies are key to understanding galaxy and structure formation in the universe.

The space density and physical properties of massive galaxies provide direct tests of galaxy formation scenarios in the context of Cold Dark Matter (CDM) structure formation models. These models predict galaxies assemble through mergers of dark matter haloes over time (Springel et al. [28], Cole et al. [29], Kauffmann et al. [30]). Since the universe is not old enough at $z \simeq 5$ for many mergers to occur, the mass in large galaxies is closely related to the mass in its initial dark matter halo. The distribution of initial halo masses is strongly constrained by the Cosmic Microwave Background (CMB) measurements (Bennett et al. [31]). So, the mass function of very massive galaxies at $z \simeq 5$ is also strongly constrained. As a result, the mass function and clustering of the LAEs and LBGs at $z \simeq 5$ provide a key test of the CDM models.

The present data on the $z \simeq 5$ mass function suggest more high mass galaxies at $z \simeq 5$ than predicted by most variants of CDM models (Eyles et al. [24, 25], Yan et al. $[26,15])$. We will dramatically improve these measurements by constraining the density of bright, and hence very high mass objects (those with magnitudes of $z^{\prime}<25.5$ ), increasing the present sample size by a factor of 100 . The environmental dependence of the galaxy mass function at $z=5.7$ and $z=4.95$ will also constrain CDM models, which predict the largest galaxies should fall in high density regions (Springel et al. [28]). The LAEs and LBGs in the COSMOS field are the only sample allowing such studies.

Time resolved 3.6 and $4.5 \mu \mathrm{m}$ observations of the about 3000 AGN contained in the COSMOS field will allow to probe variability on time scales of minutes to years. The existence of such variability is already indicated by ground-based observations; however, only for a very small sample of sources. Systematic studies will lead to an increasing insight to the physical processes responsible for this variability (e.g., inhomogeneities in the medium along the line of sight, encountered by relativistic outflow or small scale instabilities in the accretion disk). 


\subsection{Photometric Measurement of Physical Properties of Galaxies}

The stellar mass of a galaxy is traced by long lived red stars, which dominate the integrated light of galaxies at rest-frame optical/near-infrared wavelengths. While active star formation is dominated by short lived blue stars, which dominate the integrated light at rest frame ultraviolet (UV) wavelengths. As a result, a sensitive indicator of galaxy age and integrated stellar mass is the strength of the Balmer break at $4000 \AA$. The size of the Balmer break is directly proportional to the age of the stellar population (i.e. starburst vs. post-starburst) (Yan et al. [26], Bruzual and Charlot [32]) . Hence, three critical pieces of information are needed to study the mass and age of galaxies. These are: redshifts to constrain the rest frame position of the $4000 \AA$ break, a rest frame optical flux which is related to the stellar mass, and the rest frame ultraviolet to optical flux ratio, that indicates the age of the stellar population.

At the redshifts of the galaxies in this study, the Balmer break lies between $2.2 \mu \mathrm{m}$ and $3 \mu \mathrm{m}$. Hence, the proposed IRAC data along with ground based $z^{\prime}, J$, and $K_{S}$ data (and NICMOS F160W data in some cases) provide the rest-frame ultraviolet fluxes needed to constrain ages, dust content and star formation rates. The existing S-COSMOS (Sanders et al. [2]) Spitzer/IRAC data are not deep enough to probe the knee of the luminosity function which lies at $z^{\prime} \simeq 25$ (Iwata et al. [18]). The proposed data will allow us to probe 1.5 magnitudes farther down the luminosity function than the S-COSMOS data to $z^{\prime}=25.5$. This is 0.5 magnitudes fainter than $\mathrm{L}_{*}$ at $z=5$. A stacking analysis of the existing IRAC observations indicated galaxies at $z^{\prime} \simeq 25.5$ have an IRAC flux of $\sim 0.3 \mu \mathrm{Jy}\left(m_{3.6} \simeq 25.2 \mathrm{AB}\right.$ mag), which is a factor of 3 deeper than the existing IRAC observations (Sanders et al. [2]).

The COSMOS team has extensive knowledge of the multi-wavelength data which is critical to the maximal effectiveness of the deep IRAC imaging - both the IRAC observations planning, image reduction and mosaicing, PSF matching and source extraction and photometry. Extremely critical also, is the extensive knowledge of the the other COSMOS photometric datasets and the estimation and validation of photometric redshifts and galaxy properties from these diverse data.

\section{THE LEGACY OF COSMOS}

The COSMOS field is the largest contiguous area ever imaged by HST; Spitzer and Chandra have also devoted some of their largest ever allocations to this survey. Being equatorial and accessible to all telescopes (in both hemispheres), it is likely to remain the most thoroughly observed extragalactic field well into the JWST era. As such, it is destined to represent the reference field for future studies of observational cosmology, attracting massive time investments by every new facility coming on line, e.g., Herschel, ALMA and JWST. (It is already a prime target for Hershel GTO.)

COSMOS builds on the earlier, pioneering cosmic evolution studies (HDF, GOODS, GEMS, AEGIS and UDF) by probing environmental dependences on all scales of cosmic large-scale structure at the epochs of maximum activity (with a large sample volume to reduce cosmic variance). Over 2 million galaxies and AGN are detected in the 2 square degree field. Our most recent photometric redshifts derived from 20 
bands (Fig. 1) and with deeper IR photometry are now yielding $\sigma_{z} /(1+z)<0.02$ out to $z \sim 2.5$ with a sample of $\sim 800,000$ galaxies (see Fig. 1)! Photometric masses are also obtained for over a million galaxies from the ground-based and Spitzer-IRAC imaging. The growth of galaxies, AGN and dark matter can be traced by COSMOS over $\sim 75 \%$ of the age of the universe.

The COSMOS (and the deeper but smaller GOODS) surveys are the backbones to planning of observational programs for future space missions such as JWST. A critical feature of these surveys is the combination of data from many facilities, across the electromagnetic spectrum for a comprehensive investigation of cosmic evolution. By doing so on such a highly statistically significant sample (by virtue of their size and homogeneous data quality), investigators can test theories on a much stricter quantitative basis.

\section{REFERENCES}

1. Scoville, N. et al., 2007, ApJS, 172,1

2. Sanders, D. et al., 2007, ApJS, 172, 86

3. Hasinger, G. et al., 2007, ApJS 172, 29

4. Taniguchi, Y. et al., 2007, ApJS, 172,9

5. Mobasher et al., 2007, ApJS, 172, 17

6. Lilly, S. 2007 et al., 2007 , ApJS, 172, 70

7. Capak, P. et al., 2007, ApJS, 172, 99

8. Massey, R et al., 2007, Nature, 445,286

9. Scoville, N. et al., 2007, ApJS, 172,150

10. Guzzo, L. et al., 2007, ApJS, 172, 254

11. Finoguenov, A. et al., 2007, ApJS, 172, 182

12. Capak, P. et al., 2007, ApJS, 172, 284

13. Haiman, Z. and Loeb, A., 1999, ApJ, 519, 479

14. Yahata et al.,, 2000, ApJ, 538, 493

15. Yan et al., 2006, ApJ, 651,24

16. Steidel, C et al., 2003 ApJ, 592, 728

17. Steidel, C et al., 1999 ApJ, 519, 1

18. Iwata, I. et al., 2007, MNRAS, 376, 1557

19. Hu, E., and Cowie, L., 2006, Nature, 440, 1145

20. Hu, E et al., 2004, AJ, 127, 563

21. Ouchi, M. et al., 2005, ApJL, 620, L1

22. Murayama, T., 2007, ApJS, 172, 523

23. Mobasher, B. et al., 2005, ApJ, 635, 832

24. Eyles, L. et al., 2005, MNRAS, 364, 443

25. Eyles, L. et al., 2007, MNRAS, 374, 910

26. Yan, $\mathrm{H}$ et al., 2005, ApJ, 634, 109

27. Lai, K. et al., 2007, ApJ, 655, 704

28. Springel, V., 2005, Nature, 435, 629

29. Cole, D. et al., 2000, MNRAS, 319,168

30. Kauffmann, G et al., 1993, MNRAS, 264, 201

31. Bennett, C., 2003, ApJS, 148, 97

32. Bruzual, G. and Charlot, S., 2003, MNRAS, 344, 1000 